\title{
BEHAVIOURAL THEORY FOR MOBILE AMBIENTS
}

\author{
MassimoMerro \\ Dipartimento di Informatica, Università di Verona, Italy \\ Francesco Zappa Nardelli \\ INRIA \& Computer Laboratory, University of Cambridge, UK
}

\begin{abstract}
We study the behavioural theory of Cardelli and Gordon's Mobile Ambients, by focusing on a standard contextual equivalence, reduction barbed congruence. We prove a context lemma that allows the derivation of contextual equivalences by considering only contexts for concurrency and locality. We go further and give a characterisation of reduction barbed congruence over arbitrary processes in terms of a labelled bisimilarity defined over a restricted class of processes, called systems. This characterisation extends and completes an earlier result on bisimulation proof methods for Mobile Ambients, that was restricted to systems. The characterisation is then used to prove a collection of algebraic laws.
\end{abstract}

\section{Introduction}

The calculus of Mobile Ambients [5], abbreviated MA, has been introduced as a process calculus for describing mobile agents. In MA, the term $n[P]$ represents an agent, or ambient, named $n$, executing the code $P$. The ambient $n$ is a bounded, protected, and (potentially) mobile space where the computation $P$ takes place. In turn $P$ may contain other ambients, may perform (local) communications, or may exercise capabilities, which allow entry to or exit from named ambients. Ambient names, such as $n$, are used to control access to the ambient's computation space and may be dynamically created as in the $\pi$-calculus, [17], using the construct $(\boldsymbol{\nu} n) P$. A system in MA is a collection of ambients running in parallel, where the knowledge of certain names may be restricted.

Background Reduction barbed congruence [13] is a coinductive contextual relation widely adopted as a natural behavioural equivalence for process languages. It is defined as the largest equivalence relation that (i) is a congruence; (ii) preserves, in some sense, the reduction semantics of the language; (iii) preserves barbs, some simple observational property of terms. Reduction barbed congruence can be virtually applied to any process language, but the universal quantification over all contexts often represents a serious obstacle in proofs. Simpler proof techniques are traditionally based on labelled bisimilarities, which do not involve context quantification. 
The work [16] focuses on MA systems, and its main result is the definition of a labelled transition system (LTS) and of a labelled bisimilarity over systems (denoted $\approx$ ) that coincides with reduction barbed congruence over systems (denoted $\cong_{8}$ ). The paper [16] also provide up-to context and up-to expansion proof techniques, which are used to prove a set of algebraic laws between systems. Those laws allows us to equate bigger systems starting from equivalent sub-systems. However, they cannot be used to equate processes starting from equivalent sub-processes.

The main reason why the paper [16] focuses on systems rather than processes is the fact that MA semantics suffers a phenomenon called stuttering, originated by ambients that may repeatedly enter and exit another ambient. In fact, as observed in [18], the two processes

$$
P \stackrel{\text { def }}{=} \text { in_n.out_n.in_n.R and } Q \stackrel{\text { def }}{=} \text { in_n.out_n.in_n.R+in_n.R }
$$

are not distinguished by reduction barbed congruence overprocesses $\left(\right.$ denoted $\left.\cong_{p}\right){ }^{1}$ Intuitively, a context can provide an ambient named $n$, that originates the reduction: $k[Q] \mid n[] \rightarrow n[k[R]]$. But the process $P$ can match it performing three consecutive reductions: $k[P] \mid n[] \rightarrow \rightarrow \rightarrow n[k[R]]$. As stuttering cannot be observed, a labelled bisimilarity that successfully captures $\cong_{p}$ must be insensitive to stuttering. Characterising the interactions that a process has with its environment in a way insensitive to stuttering would make the definition of the LTS (and of the corresponding weak actions) quite complicated. Focusing on systems leads to definition of a simple and natural LTS; stuttering is then modelled in terms of standard weak actions.

Systems represent an expressive subset of MA processes, but the results of [16] only guarantee that the equivalence is preserved by system contexts (a subset of the static contexts). In particular, they leave open the question if the bisimulation proof methods are sound when all MA contexts are considered. Also, a proper treatment of processes is needed to fully understand the subtleties of MA algebraic theory.

Contributions This paper develops a behavioural theory of MA processes, extending and completing the results of [16]. The main contributions are:

- a context lemma for reduction barbed congruence over processes, that allows the derivation of contextual equivalences by considering only parallel composition and ambient nesting;

- a characterisation of reduction barbed congruence over processes in terms of a simple relation based on the bisimilarity over systems introduced in [16]. In particular, we show that $\cong_{\mathrm{p}}$ coincides with the relation $\mathcal{S}$ below, defined on top of $\approx$ :

$$
\mathcal{S} \stackrel{\text { def }}{=}\{(P, Q): k[P \mid R] \approx k[Q \mid R] \text { for all } k \text { and } R\} .
$$

This result, combined with the up-to proof techniques for the labelled bisimilarity, gives an effective proof method to show the behavioural equality of two processes;

- we show that when comparing two systems, the labelled bisimilarity $\approx$ is a sound and complete technique for reduction barbed congruence over processes.

\footnotetext{
${ }^{1}$ For simplicity we use external choice à la CCS; the same phenomenon can be exhibited using replication.
} 
As a consequence, all the algebraic laws given in [16] hold for reduction barbed congruence over processes;

- we extend our results to the calculus equipped with asynchronous communication of capabilities, and for the first time we give a complete semantic treatment of communication in MA;

- we prove a collection of algebraic laws on processes to be added to the laws on systems inherited from [16].

We believe that the proofs of the two main results of the paper, i.e. the context lemma (Theorem 9) and the labelled characterisation of reduction barbed congruence over processes (Theorem 10) are non-standard and interesting in their own. In fact, the proof of Theorem 10 requires sophisticated up-to proof techniques, whereas the proof of Theorem 9 relies on the characterisation of reduction barbed equivalence given in Theorem 10: a direct proof seems difficult.

Related work Higher-order LTSs for Mobile Ambients can be found in [4, 9, 20, 7]. But we are not aware of any form of bisimilarity defined using these LTSs. A simple first-order LTS for MA without restriction is proposed by Sangiorgi in [18]. Using this LTS the author defines an intensional bisimilarity for MA that separates terms on the basis of their internal structure. Other forms of labelled bisimilarity for higherorder distributed calculi, such as Safe Ambients [14], Distributed $\pi$-calculus [12], Safe $\mathrm{D} \pi$ [11] Seal [21], Nomadic Pict [19], a Calculus for Mobile Resources [8], can be found in $[15,10,6,19,8,3,11]$, but only $[15,10,8,3,11]$ prove labelled characterisations of a contextually defined notion of equivalence. In [9], Gordon and Cardelli give a context lemma for a Morris-style contextual equivalence. However, unlike ours, their context lemma closes the contextual equivalence also under restriction. In that paper, using the context lemma, the authors prove the correctness of the perfect firewall equation.

Outline In Section 2 we recall the definitions and results of [16]. In Section 3 we focus on processes, and we prove several properties of reduction barbed congruence over processes, including its characterisation in terms of the labelled bisimilarity over systems. In Section 4 we extend the results of the previous section to the full calculus with communication. Finally, in Section 5 we use our proof methods to prove a collection of algebraic laws.

\section{A semantic theory for systems}

Syntax and reduction semantics In Table 1 we report the syntax of MA processes, where $\mathbf{N}$ denotes an infinite set of names, ranged over by $n, h, \ldots$ We also define a subset of MA processes, called systems.

The syntax for processes is standard, [5], except for replication that is replaced by replicated prefixing, !C.P. As in the $\pi$-calculus, this simplifies the definition of the LTS. A system is a collection of ambients running in parallel, where the knowledge of certain ambient names may be restricted among two or more ambients.

We use a number of notational conventions. Parallel composition has the lowest precedence among the operators. The process $\boldsymbol{C} . C^{\prime} . P$ is read as $\boldsymbol{C} .\left(C^{\prime} . P\right)$. We omit trailing dead processes, writing $C$ for $C . \mathbf{0}$, and $\boldsymbol{n}[]$ for $\boldsymbol{n}[\mathbf{0}]$. Restriction $(\boldsymbol{\nu} n) P$ acts as binder for name $\boldsymbol{n}$, and the set of free names of $P, \mathrm{fn}(\mathrm{P})$, is defined accordingly. 


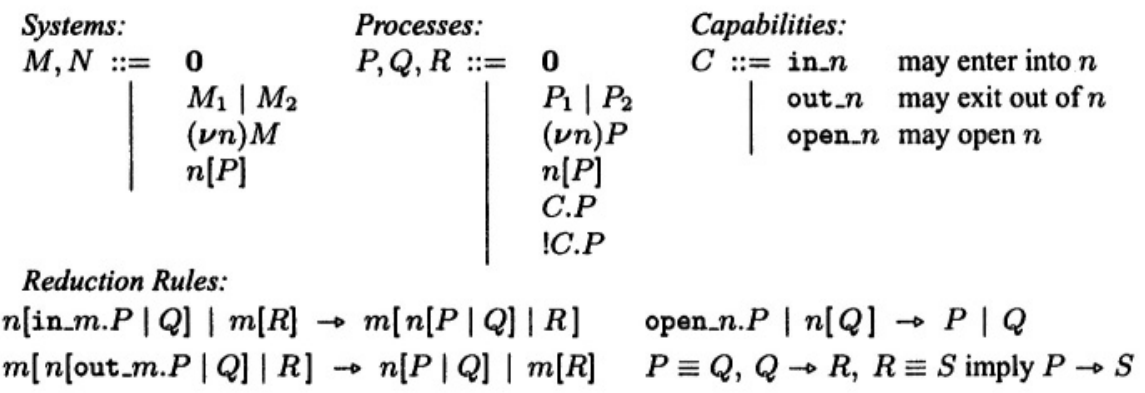

\section{Table 1. The Mobile Ambients in Two Levels: Syntax and Reduction Rules}

A context is a process not completely defined; we write - for the hole. A static context is a context where the hole does not appear underneath prefix and replication. The dynamics of the calculus is specified by a reduction relation, $\rightarrow$, which is the least relation over processes closed under static contexts and satisfying the rules in Table 1. As systems are processes with a special structure, the rules of Table 1 also describe the evolution of systems. The reduction semantics relies on an auxiliary relation called structural congruence, $\equiv$, that brings the participants of a potential interaction into contiguous positions. Its definition is standard (see [5]). It is easy to check that systems always reduce to systems.

The behavioural theory of systems We focus on a generalisation of the reduction barbed congruence, a contextual, reduction closed, and barb preserving equivalence relation. We now explain what these properties mean.

A system context is a context generated by the following grammar:

$C[-]::=-|C[-]| M|M| C[-]|(\boldsymbol{\nu}) C[-]| n[C[-] \mid P] \mid n[P \mid C[-]]$ where $M$ is an arbitrary system, and $P$ is an arbitrary process. A relation $\mathcal{R}$ over systems is preserved by system contexts if $M \mathcal{R} N$ implies $C[M] \mathcal{R} C[N]$ for all system contexts $C[-]$.

A relation $\mathcal{R}$ is reduction closed if whenever $P \mathcal{R} Q$ and $P \rightarrow P^{\prime}$ there is some $Q^{\prime}$ such that $Q \rightarrow \rightarrow^{*} Q^{\prime}$ and $P^{\prime} \mathcal{R} Q^{\prime}$, where $\rightarrow{ }^{*}$ denotes the reflexive and transitive closure of $\rightarrow$.

In MA the observation predicate $P \downarrow_{n}$ denotes the possibility of the process $P$ interacting with the environment via the ambient $n$. We write $P \downarrow_{n}$ if $P \equiv(\nu \tilde{m})\left(n\left[P_{1}\right] \mid\right.$ $\left.P_{2}\right)$ with $n \notin\{\tilde{m}\}$. We write $P \Downarrow_{n}$ if there exists $P^{\prime}$ such that $P \rightarrow \rightarrow^{*} P^{\prime}$ and $P^{\prime} \downarrow_{n}$. A relation $\mathcal{R}$ is barb preserving if $P \mathcal{R} Q$ and $P \downarrow_{n}$ imply $Q \Downarrow_{n}$.

Definition 1 (Reduction barbed congruence over systems) Reduction barbed congruence over systems, written $\cong_{\mathbf{8}}$ is the largest symmetric relation over systems which is reduction closed, barb preserving, and preserved by system contexts.

We briefly summarise the results of [16]. The main result is the definition of a LTS and of a labelled bisimilarity over systems that coincide with reduction barbed congruence over systems. The LTS is defined over processes, although the labelled bisimilarity only considers actions going from systems to systems. A distinction is made between pre-actions and env-actions: the former denote the possibility to exercise certain capabilities whereas the latter model the interaction of a system with its 
Pre-actions: $\pi::=$ in_n | out_n | open_n | enter_n | amb_n | exit_n Env-actions: $\mu$ ::= k.enter_n | k.exit_n | *.enter_n | *.exit_n $n . \overline{\text { enter_ }} k \mid k$.open_n

Actions: $\quad \alpha::=\mu \mid \tau$

Outcomes: $O::=P \mid K \quad$ Concretions: $K::=(\nu \tilde{m})\langle P\rangle Q$

Table 2. Pre-actions, Env-actions, Actions, Concretions, and Outcomes

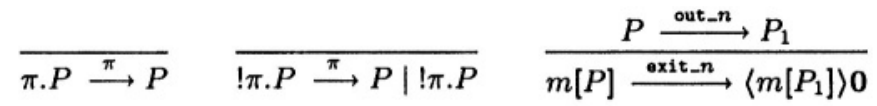

$$
\begin{aligned}
& \frac{P \stackrel{\text { in_ } n}{\longrightarrow} P_{1}}{n[P] \stackrel{\text { amb_n }}{\longrightarrow}\langle P\rangle 0} \quad \frac{P \stackrel{\pi}{\longrightarrow} O \quad n \notin \mathrm{fn}(\pi)}{m[P] \stackrel{\text { enter_ } n}{\longrightarrow}\left\langle m\left[P_{1}\right]\right\rangle 0} \quad \frac{\pi}{(\nu n) P} \stackrel{(\nu n) O}{\longrightarrow} \\
& \frac{P \stackrel{\pi}{\longrightarrow} O}{P|Q \stackrel{\pi}{\longrightarrow} O| Q} \\
& Q|P \stackrel{\pi}{\longrightarrow} Q| O
\end{aligned}
$$

Table 3. Labelled Transition System - Pre-actions

$$
\begin{aligned}
& \frac{P \stackrel{\text { enter_n }}{\longrightarrow}(\nu \tilde{p})\left\langle P_{1}\right\rangle P_{2} \quad Q \stackrel{\text { amb_n }}{\longrightarrow}(\nu \tilde{q})\left\langle Q_{1}\right\rangle Q_{2}{ }^{(*)}}{P \mid Q \stackrel{\tau}{\longrightarrow}(\nu \tilde{p})(\nu \tilde{q})\left(n\left[P_{1} \mid Q_{1}\right]\left|P_{2}\right| Q_{2}\right)} \\
& Q\left|P \stackrel{\tau}{\longrightarrow}(\nu \tilde{q})(\nu \tilde{p})\left(n\left[Q_{1} \mid P_{1}\right]\left|Q_{2}\right| P_{2}\right) \quad Q\right| P \stackrel{\tau}{\longrightarrow} Q \mid P^{\prime} \\
& \frac{P \stackrel{\text { exit_n }}{\longrightarrow}(\nu \tilde{m})\left\langle k\left[P_{1}\right]\right\rangle P_{2}}{n[P] \stackrel{\tau}{\longrightarrow}(\nu \tilde{m})\left(k\left[P_{1}\right] \mid n\left[P_{2}\right]\right)} \\
& \frac{P \stackrel{\text { open_n }}{\longrightarrow} P_{1} Q \stackrel{\text { amb_n } n}{\longrightarrow}(\nu \tilde{m})\left\langle Q_{1}\right\rangle Q_{2}}{P\left|Q \stackrel{\tau}{\longrightarrow} P_{1}\right|(\nu \tilde{m})\left(Q_{1} \mid Q_{2}\right)} \\
& Q\left|P \stackrel{\tau}{\longrightarrow}(\nu \tilde{m})\left(Q_{1} \mid Q_{2}\right)\right| P_{1} \\
& \begin{array}{l}
\frac{P \stackrel{\tau}{\longrightarrow} P^{\prime}}{P\left|Q \stackrel{\tau}{\longrightarrow} P^{\prime}\right| Q} \\
Q|P \stackrel{\tau}{\longrightarrow} Q| P^{\prime} \\
\frac{P \stackrel{\tau}{\longrightarrow} Q}{n[P] \stackrel{\tau}{\longrightarrow} n[Q]}
\end{array} \\
& \frac{P \stackrel{\tau}{\longrightarrow} P^{\prime}}{(\nu n) P \stackrel{\tau}{\longrightarrow}(\nu n) P^{\prime}}
\end{aligned}
$$

$\left(^{*}\right)$ We require $\left(\mathrm{fn}\left(P_{1}\right) \cup \mathrm{fn}\left(P_{2}\right)\right) \cap\{\tilde{q}\}=\left(\mathrm{fn}\left(Q_{1}\right) \cup \mathrm{fn}\left(Q_{2}\right)\right) \cap\{\tilde{p}\}=\emptyset$

Table 4. Labelled Transition System - $T$-actions

$$
\begin{aligned}
& \frac{P \stackrel{\text { enter_ } n}{\longrightarrow}(\nu \tilde{m})\left\langle k\left[P_{1}\right]\right\rangle P_{2}{ }^{(\dagger)}}{P \stackrel{k . \text { enter_ } n}{\longrightarrow}(\nu \tilde{m})\left(n\left[k\left[P_{1}\right] \mid \text { ㅇ }\right] \mid P_{2}\right)} \\
& \frac{P \stackrel{\text { amb_ } n}{\longrightarrow}(\nu \tilde{m})\left\langle P_{1}\right\rangle P_{2}{ }^{(\dagger)}}{P \stackrel{n . \overline{\text { nnter }}_{-} k}{\longrightarrow}(\nu \tilde{m})\left(n\left[P_{1} \mid k[\circ]\right] \mid P_{2}\right)} \\
& \frac{P \stackrel{\text { enter_ } n}{\longrightarrow}(\nu \tilde{m})\left\langle k\left[P_{1}\right]\right\rangle P_{2}^{(\ddagger)}}{P \stackrel{\text { *.enter_ } n}{\longrightarrow}(\nu \tilde{m})\left(n\left[k\left[P_{1}\right] \mid \text { o] } \mid P_{2}\right)\right.}
\end{aligned}
$$$$
\frac{P \stackrel{\text { exit_n }}{\longrightarrow}(\nu \tilde{m})\left\langle k\left[P_{1}\right]\right\rangle P_{2}{ }^{(\dagger)}}{P \stackrel{k . \text { exit_ } n}{\longrightarrow}(\nu \tilde{m})\left(k\left[P_{1}\right] \mid n\left[\circ \mid P_{2}\right]\right)}
$$$$
\frac{P \stackrel{\text { amb_ } n}{\longrightarrow}(\nu \tilde{m})\left\langle P_{1}\right\rangle P_{2}}{P \stackrel{k . \text { open_ } n}{\longrightarrow} k\left[\circ \mid(\nu \tilde{m})\left(P_{1} \mid P_{2}\right)\right]}
$$$$
\frac{P \stackrel{\text { exit_n }}{\longrightarrow}(\nu \tilde{m})\left\langle k\left[P_{1}\right]\right\rangle P_{2}{ }^{(\ddagger)}}{P \stackrel{\text { *.exit_n }}{\longrightarrow}(\nu \tilde{m})\left(k\left[P_{1}\right] \mid n\left[\circ \mid P_{2}\right]\right)}
$$

(†) We require $k \notin \tilde{m}$. (†) We require $k \neq n$ and $k \in \tilde{m}$

Table 5. Labelled Transition System - Env-actions 
environment. Internal computations are modelled by $\tau$-actions. Only env-actions and $\tau$-actions model the evolution of a system at run-time.

The pre-actions, defined in Table 3, are of the form $P \stackrel{\pi}{\longrightarrow} O$ where the ranges of $\pi$ and of $O$, the outcomes, are reported in Table 2. An outcome is a process $Q$, if $\pi$ is a prefix of the language, or a concretion, of the form $(\nu \tilde{m})\langle P\rangle Q$, when an ambient boundary is somehow involved. In this case, intuitively, $P$ represents the part of the system affected by the action while $Q$ is not, and $\tilde{m}$ is the set of private names shared by $P$ and $Q$. We adopt the convention that if $K$ is the concretion $(\boldsymbol{\nu} \tilde{m})\langle P\rangle Q$, then $(\boldsymbol{\nu} r) K$ is a shorthand for $(\boldsymbol{\nu} \tilde{m})\langle P\rangle(\boldsymbol{\nu} r) Q$, if $r \notin \mathrm{fn}(P)$, and for the concretion $(\boldsymbol{\nu} \tilde{m} \tilde{m})\langle P\rangle Q$ otherwise. In the rule ( $\pi$ Par) we define $K \mid R$ to be the concretion $(\boldsymbol{\nu} \tilde{m})\langle P\rangle(Q \mid R)$, where $\tilde{m}$ are chosen, using $\alpha$-conversion if necessary, so that $\mathrm{fn}(R) \cap\{\tilde{m}\}=\emptyset$. Similarly for $R \mid K$. Finally, $(\nu \tilde{m})\langle P\rangle(\mathbf{0} \mid R)$ is abbreviated by $(\boldsymbol{\nu} \tilde{m})\langle P\rangle R$.

The $\tau$-actions, defined in Table 4 , model the internal evolution of processes. It can be shown that if $P \stackrel{\tau}{\longrightarrow} P^{\prime}$ then $P \rightarrow P^{\prime}$; conversely if $P \rightarrow P^{\prime}$ then $P \stackrel{\tau}{\longrightarrow} \equiv P^{\prime}$.

The env-actions, defined in Table 5, are of the form $M \stackrel{\mu}{\longrightarrow} M^{\prime}$, where the range of $\mu$ is given in Table 2. Roughly speaking, env-actions capture the interaction of a system with its environment. In practice, env-actions turn concretions into running systems by explicitly introducing the environment's ambient interacting with the process being considered. The content of this ambient is arbitrary; it is left unspecified in the LTS and is taken into account only in the definition of bisimulation. For convenience, the syntax of processes is extended with a special process o, used to pinpoint those ambients whose content must be instantiated in the bisimulation. Operationally, the process $\circ$ is analogous to the inactive process (it is simply a placeholder). Unlike pre-actions and $\tau$-actions, env-actions do not have structural rules; this is because env-actions are supposed to be performed by systems that can directly interact with the environment.

The set of the actions is the set of env-actions extended with $\tau$. As env-ctions capture the interaction of a system with its environment, the definition of bisimilation only takes into account actions (and not pre-actions). More explanations on the LTS can be found in [16].

The definition of weak actions is standard: $\Rightarrow$ denotes the reflexive and transitive closure of $\stackrel{\tau}{\longrightarrow}$; $\stackrel{\alpha}{\longrightarrow}$ denotes $\Rightarrow \stackrel{\alpha}{\longrightarrow} \Rightarrow: \stackrel{\alpha}{\Longrightarrow}$ denotes $\Rightarrow$ if $\alpha=\tau$ and $\stackrel{\alpha}{\Longrightarrow}$ otherwise.

Env-actions introduce a special process o to pinpoint those ambients whose content will be specified in the bisimilarity. The $\bullet$ operator instantiates the placeholder with a process.

Definition 2 Let $T$ and $T_{i}$ be either systems or processes. Then, for a process $P$, we define:

$$
\begin{gathered}
0 \bullet P \stackrel{\text { def }}{=} 0 \quad\left(T_{1} \mid T_{2}\right) \bullet P \stackrel{\text { def }}{=}\left(T_{1} \bullet P\right) \mid\left(T_{2} \bullet P\right) \quad \circ \bullet P \stackrel{\text { def }}{=} P \\
n[R] \bullet P \stackrel{\text { def }}{=} n[R \bullet P] \quad(\nu n) T \bullet P \stackrel{\text { def }}{=}(\nu n)(T \bullet P) \text { if } n \notin \operatorname{fn}(P) \\
|C . R \bullet P \stackrel{\text { def }}{=}| C .(R \bullet P) \quad C . R \bullet P \stackrel{\text { def }}{=} C .(R \bullet P)
\end{gathered}
$$

Bisimilarity over systems is defined below. 
Definition 3 (Bisimilarity) A symmetric relation $\mathcal{R}$ is a bisimulation if $M \mathcal{R} N$ implies:

- if $M \stackrel{\alpha}{\rightarrow} M^{\prime}, \alpha \notin\left\{*\right.$. enter_n,*.exit_n\}, then there is a system $N^{\prime}$ such that $N \stackrel{\hat{\alpha}}{\Rightarrow} N^{\prime}$ and for all processes $P$ it holds $M^{\prime} \bullet P \mathcal{R} N^{\prime} \bullet P$;

- if $M \stackrel{\text { *.ontor_n }}{\longrightarrow} M^{\prime}$ then there is a system $N^{\prime}$ such that $N \mid n[0] \Rightarrow N^{\prime}$ and for all processes $P$ it holds $M^{\prime} \bullet P \mathcal{R} N^{\prime} \bullet P$;

- if $M \stackrel{\text { *.oxit_n } n}{\longrightarrow} M^{\prime}$ then there is a system $N^{\prime}$ such that $n[0 \mid N] \Rightarrow N^{\prime}$ and for all processes $P$ it holds $M^{\prime} \bullet P \mathcal{R} N^{\prime} \bullet P$.

Systems $M$ and $N$ are bisimilar, denoted $M \approx N$, if $M \mathcal{R} N$ for a bisimulation $\mathcal{R}$.

Theorem 4 Reduction barbed congruence over systems and bisimilarity coincide.

We end this section with a sound up-to proof technique for bisimilarity that generalises those presented in [16]. The expansion [2], written $\gtrsim$, is an asymmetric variant of $\approx$ such that $M \gtrsim N$ holds if $M \approx N$ and $M$ has at least as many $\tau$-moves as $N$.

Definition 5 (Bisimulation up to context and up to $\gtrsim \approx$ ) A symmetric relation $\mathcal{R}$ over systems is a bisimulation up to context and up to $\gtrsim \approx$ if $M \mathcal{R} N$ implies:

- if $M \stackrel{\alpha}{\longrightarrow} M^{\prime \prime}, \alpha \notin\left\{* . e n t e r \_n, * . e x i t \_n\right\}$, then there exists a system $N^{\prime \prime}$ such that $N \stackrel{\hat{\alpha}}{\Longrightarrow} N^{\prime \prime}$, and for all processes $P$ there is a system context $C[-]$ and systems $M^{\prime}$ and $N^{\prime}$ such that $M^{\prime \prime} \bullet P \gtrsim C\left[M^{\prime}\right], N^{\prime \prime} \bullet P \approx C\left[N^{\prime}\right]$, and $M^{\prime} \mathcal{R} N^{\prime}$;

- if $M \stackrel{\text { *.ontor_n }}{\longrightarrow} M^{\prime \prime}$ then there exists a system $N^{\prime \prime}$ such that $N \mid n[0] \Rightarrow N^{\prime \prime}$, and for all processes $P$ there is a system context $C[-]$ and systems $M^{\prime}$ and $N^{\prime}$ such that $M^{\prime \prime} \bullet P \gtrsim C\left[M^{\prime}\right], N^{\prime \prime} \bullet P \approx C\left[N^{\prime}\right]$, and $M^{\prime} \mathcal{R} N^{\prime}$;

- if $M \stackrel{\text { *.oxit_n }}{\longrightarrow} M^{\prime \prime}$ then there exist a system $N^{\prime \prime}$ such that $n[0 \mid N] \Rightarrow N^{\prime \prime}$, and for all processes $P$ there is a system context $C[-]$ and systems $M^{\prime}$ and $N^{\prime}$ such that $M^{\prime \prime} \bullet P \gtrsim C\left[M^{\prime}\right], N^{\prime \prime} \bullet P \approx C\left[N^{\prime}\right]$, and $M^{\prime} \mathcal{R} N^{\prime}$.

Theorem 6 If $\mathcal{R}$ is a bisimulation up to context and up to $\approx \approx$ then $\mathcal{R} \subseteq \approx$.

\section{A semantic theory for processes}

In this section we characterise reduction barbed congruence over processes.

Definition 7 Reduction barbed congruence over processes, written $\cong_{p}$, is the largest symmetric relation over processes which is reduction closed, barb preserving, and preserved by arbitrary single-hole contexts.

In the definition above, the universal quantification over arbitrary contexts makes direct proofs of the equivalence of two systems difficult and error prone. Reducing the number of contexts to consider in the quantification is a first step towards the definition of a useful proof technique, and, broadly speaking, towards an understanding of the algebraic theory of processes.

We show that it is possible to work with a lighter definition of contextuality. In particular it suffices to close the contextual equivalence only under the two crucial constructions of MA: parallel composition (to model concurrency) and ambient nesting (to model locality). 
Definition 8 Reduction barbed equivalence over processes, written $\cong_{\mathbf{p}}^{\mathbf{e}}$, is the largest symmetric relation over processes which is reduction closed, barb preserving, and closed underparallel composition and ambient construct.

Theorem 9 (Context Lemma) The relations $\cong_{\mathbf{p}}$ and $\cong_{\mathbf{p}}^{\mathbf{e}}$ coincide.

Reduction barbed equivalence over processes still requires us to consider non-trivial contexts. More than that, a direct proof of the context lemma is surprisingly difficult. We look for a more operative characterisation of $\cong \mathbf{p}$, and we postpone the proof of the context lemma after Theorem 10.

Theorem 10 (Characterisation of $\cong$ pet

$$
\mathcal{S}=\{(P, Q): k[P \mid R] \approx k[Q \mid R], \text { for all } k, R\} .
$$

The relations $\cong_{\mathbf{p}}^{\mathbf{e}}$ and $\mathcal{S}$ coincide.

To prove Theorem 10 we need some technical lemmas. The next two lemmas are necessary for proving the completeness part of Theorem 10. In particular Lemma 11 says that reduction barbed equivalence over processes is preserved by restriction. This result will be also useful when proving the context lemma.

Lemma 11 If $P \cong_{\mathrm{p}}^{\mathrm{e}} Q$, then $(\nu n) P \cong_{\mathrm{p}}^{\mathrm{e}}(\boldsymbol{\nu} n) Q$.

Lemma $12 \cong 0$

Everything is now in place to prove Theorem 10. In the remainder of the paper, when working with a relation $\mathcal{R}$ over processes and/or systems, we denote $\mathcal{R}^{=}$the symmetric closure of $\mathcal{R}$.

Proof of Theorem 10. We first prove that $P \simeq_{\mathrm{p}}^{0} Q$ implies $P \mathcal{S} Q$. For that, we must show that for all $k, R$, it holds $k[P \mid R] \approx k[Q \mid R]$. Both $k[P \mid R]$ and $k[Q \mid R]$ are systems, and it holds $k[P \mid R] \cong \cong_{\mathrm{p}}^{\mathfrak{e}} k[\boldsymbol{Q} \mid \boldsymbol{R}]$ because $\cong_{\mathrm{p}}^{\mathrm{e}}$ is closed under parallel composition and ambient construct. The result follows from Lemma 12 and Theorem 4.

It remains to prove that $\mathcal{S} \subseteq \cong$. For that, we must show that $\mathcal{S}$ is reduction closed, barb preserving, and closed under parallel composition and ambient construct.

1. $\mathcal{S}$ is reduction closed. Suppose $P \mathcal{S} Q$ and $\boldsymbol{P} \rightarrow \boldsymbol{P}^{\prime}$. Let $\boldsymbol{n}$ be a name such that $n \notin \mathrm{fn}(P, Q)$. Then, by definition of $\mathcal{S}$, it holds $n[P] \approx n[Q]$. As $n \notin \mathrm{fn}(P, Q)$, and because of the correspondence between $\tau$-transitions and reductions, there is a system $M$ such that $n[P] \stackrel{\tau}{\longrightarrow} M \equiv n\left[P^{\prime}\right]$. As $n[P] \approx n[Q]$, there is $N$ such that $n[Q] \Rightarrow N$ and $M \approx N$. But $n \notin$ fn $(P, Q)$, and there must be $Q^{\prime}$ such that $Q \rightarrow{ }^{*} Q^{\prime}$ and $N \equiv n\left[Q^{\prime}\right]$; thus $n\left[P^{\prime}\right] \approx n\left[Q^{\prime}\right]$.

Now, we prove that $P^{\prime} \cong Q^{\prime}$. By the completeness result, this suffices to conclude that $\boldsymbol{P}^{\prime} \mathcal{S} \boldsymbol{Q}^{\prime}$, as desired. The argument is by contradiction. Let us suppose that $\boldsymbol{P}^{\prime} \boldsymbol{F}_{\mathrm{p}}^{\mathrm{e}} \boldsymbol{Q}^{\prime}$. From the definition of $\simeq_{\mathrm{p}}^{\mathrm{e}}$, it follows that there is a context $C[-]$, containing only parallel composition and ambient constructs, such that at least one of the following conditions holds:

- there exists $P^{\prime \prime}$ such that $C\left[P^{\prime}\right] \rightarrow{ }^{*} P^{\prime \prime}$ and there is no $Q^{\prime \prime}$ such that $C\left[Q^{\prime}\right] \rightarrow Q^{\prime \prime}$ and $P^{\prime \prime} \cong{ }_{\mathrm{p}}^{\mathrm{e}} Q^{\prime \prime}$;

- there exists $Q^{\prime \prime}$ such that $C\left[Q^{\prime}\right] \rightarrow{ }^{*} Q^{\prime \prime}$ and there is no $P^{\prime \prime}$ such that $C\left[P^{\prime}\right] \rightarrow P^{*}$ and $Q^{\prime \prime} \cong_{\mathrm{p}}^{\mathrm{e}} P^{\prime \prime}$

- there exists $a$ such that $C\left[P^{\prime}\right] \Downarrow_{a}$ and $C\left[Q^{\prime}\right] \Downarrow_{a}$;

- there exists $a$ such that $C\left[Q^{\prime}\right\} \Downarrow_{a}$ and $C\left[P^{\prime}\right] \Downarrow_{a}$.

It is easy to verify that if one of these conditions holds, then the same condition holds also when we replace $P^{\prime}$ with $(\boldsymbol{\nu} n)\left(n\left[P^{\prime}\right] \mid\right.$ open_n) and $Q^{\prime}$ with $(\nu n)\left(n\left[Q^{\prime}\right] \mid\right.$ open_n) for $n \notin \mathrm{fn}\left(P^{\prime}, Q^{\prime}\right)$. 
For instance, in the first case there is $P^{\prime \prime}$ such that $C\left[(\nu n)\left(n\left[P^{\prime}\right] \mid\right.\right.$ open_n $\left.\left.n\right)\right] \rightarrow P^{\prime \prime}$ and there is no $Q^{\prime \prime}$ such that $C\left[(\nu n)\left(n\left[Q^{\prime}\right] \mid\right.\right.$ open_n $\left.)\right] \Rightarrow Q^{\prime \prime}$ and $P^{\prime \prime} \cong_{\mathrm{p}}^{\mathrm{e}} Q^{\prime \prime}$.

By Lemma 11, the relation $\cong_{\mathrm{p}}^{\mathbf{0}}$ is closed under restriction. So we can take the context

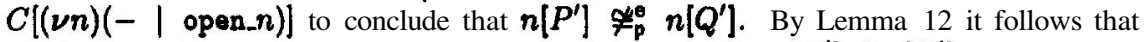
$n\left[P^{\prime}\right] \neq_{\mathbf{8}} n\left[Q^{\prime}\right]$. By Theorem 4 we reach the contradiction that $n\left[P^{\prime}\right] \not n\left[Q^{\prime}\right]$.

2. $\mathcal{S}$ is barbpreserving. Suppose that $P \mathcal{S} Q$ and $P \downarrow_{n}$. Consider the context

$$
C[-]=b[-\mid a[\text { in_n.out_n.ok[out_a.out_b }]]]
$$

where $\boldsymbol{a}, \boldsymbol{b}$ and ok are fresh for both $P$ and $Q$. Then $C[P] \approx C[Q]$ by definition of $\mathcal{S}$. As $P \ln$, the construction of $C[-]$ assures that $C[P] \Downarrow_{\text {ok. }}$. Bisimilarity is barb preserving and $C[Q] \Downarrow_{\text {ok }}$ must hold. The construction of $C[-]$ guarantees that $Q \Downarrow_{n}$.

\section{3. $\mathcal{S}$ is closed under parallel composition and ambient construct.}

$P \mathcal{S} Q$ implies $P|R \mathcal{S} Q| R$ : by definition of $\mathcal{S}$ we have $k\left[P \mid R^{\prime}\right] \approx k\left[Q \mid R^{\prime}\right]$ for all $\boldsymbol{k}, \boldsymbol{R}^{\prime}$. By taking $\boldsymbol{R}^{\prime}=\boldsymbol{R} \mid \boldsymbol{R}^{\prime \prime}$ for arbitrary $\boldsymbol{R}^{\prime \prime}$ we have $\boldsymbol{k}\left[\boldsymbol{P}|\boldsymbol{R}| \boldsymbol{R}^{\prime \prime}\right] \approx k[\boldsymbol{Q}|\boldsymbol{R}|$ $\boldsymbol{R}^{\prime \prime}$ ] for all $\boldsymbol{R}^{\prime \prime}$. This implies $P|R \mathcal{S} Q| R$.

$P \mathcal{S} Q$ implies $n[P] \mathcal{S} n[Q]$ : by definition of $\mathcal{S}$ we have $n[P] \approx n[Q]$ for all $n$. The result follows from the closure of $\approx$ under static contexts.

The characterisation of $\cong_{\mathbf{p}}^{\mathbf{e}}$ is a fundamental tool to reason about processes. As a first application, we give the proof the context lemma.

Proof of Theorem 9. For that, we have to show that $\simeq_{p}^{0}=\cong_{p}$. The inclusion $\cong_{p} \subseteq \cong_{p}^{0}$ is straightforward. For the converse we must prove that: (i) $\cong_{p}^{e}$ is reduction closed; (ii) $\cong_{p}^{e}$ is barb preserving; (iii) $\simeq_{\mathbf{p}}^{\mathbf{e}}$ is closed under arbitrary contexts. Conditions (i) and (ii) hold by definition of $\simeq_{\mathbf{p}}^{\mathbf{e}}$. It remains to show that the relation $\simeq_{\mathbf{p}}^{\mathbf{e}}$ is preserved by all process contexts. The relation $\simeq_{\mathbf{p}}^{\mathbf{e}}$ is preserved by parallel composition and ambient constructor by definition. It is also preserved by restriction by Lemma 11. It remains to prove that it is preserved by prefixing and replicated prefixing. We detail replicated prefixing.

We have to prove that if $P \cong_{\mathrm{p}}^{\mathrm{e}} Q$, then $\mid \pi . P \cong \cong_{\mathrm{p}}^{\mathrm{e}} ! \pi \cdot Q$. Rather than working directly with $\cong$, we use Theorem 10 and we prove that $! \pi \cdot P \mathcal{S} ! \pi \cdot Q$. For that, we show that $k[! \pi \cdot P \mid R] \approx$ $k[! \pi . Q \mid R]$ for all $k$ and $R$. We perform a case analysis on $\pi$, and we detail the case $\pi=\mathbf{i n} \_$. For this, we show that the relation

$$
\left.\mathcal{R}=\{(n \mid \text { in_o. } P \mid R], n[\text { lin_o. } Q \mid R]): P \approx_{\mathrm{p}}^{0} Q\right\}=U \approx
$$

is a bisimulation up to context and up to $\gtrsim \approx$. The most interesting case is when the process ! in_o.P exercises the capability in_o. Suppose

$$
n[\text { lin_o.P } \mid R] \stackrel{\text { n.ontor_o }}{\longrightarrow} \text { o[n[P|lin.o.P } \mid R] \mid \text { o] . }
$$

We have a matching transition

$$
n[\text { lin_o. } Q \mid R] \stackrel{\text { n.ontor_o }}{\longrightarrow} \text { o[n[Q } \mid \text { ! in_o. } Q \mid R] \mid \text { o] . }
$$

Since $P \cong \mathbf{p} Q$, we have $P \mathcal{S} Q$ and in turn, for all $\boldsymbol{R}^{\prime}$, we have $n\left[P \mid \boldsymbol{R}^{\prime}\right] \approx n\left[Q \mid \boldsymbol{R}^{\prime}\right]$. As $\approx$ is preserved by system contexts, for all instantiations of o it holds o[n $\left[P \mid R^{\prime}\right] \mid$ o $] \approx o[n[Q \mid$ $\left.R^{\prime}\right] \mid$ o]. By taking $R^{\prime}=$ ! in_o. $Q \mid R$, we obtain

$$
o[n[\text { lin_o.Q } Q R \mid P] \mid 0] \approx o[n[Q \mid \text { lin_o.Q } Q R] \mid 0] .
$$

Then, for all processes $S$, the following hold:

$$
\begin{aligned}
& o\left[n[P \mid \text { in_o. } P \mid R] \mid \text { o] } \bullet S \quad Z C\left[n\left[\ln \_o . P|R| P\right]\right]\right. \\
& o\left[n\left[Q\left|\operatorname{lin} \_. Q Q\right| R\right] \mid \circ\right] \bullet S \quad \approx C\left[n\left[\operatorname{lin} \_o . Q|R| P\right]\right]
\end{aligned}
$$


where $C[-]=o[-\mid S]$ (we can rearrange the terms using structural congruence because $\equiv \subseteq \gtrsim$ and $\equiv \subseteq \approx$ ). By construction of $\mathcal{R}$ we have

$$
n[\text { Iin_o.P }|R| P] R \text { n[! in.o.Q } Q R \mid P]
$$

and we can conclude that up to context and up to $\gtrsim \approx$ we are still in $\mathcal{R}$.

The result below is a consequence of Theorems 9 and 10 .

Corollary 13 The relations $\mathcal{S}$ and $\cong_{\mathrm{p}}$ coincide.

The relation $\mathcal{S}$ still involves a universal quantification over all the processes $R$. Yet, it is built on top of a labelled bisimilarity, it can be coupled with the up-to proof techniques. In turn, it reveals a useful tool to reason about processes, as illustrated by the proof of the context lemma and by the other examples given in Section 5 .

Systems, revisited In [16], we conjectured that when working with systems reduction barbed congruence over systems $\left(_{\mathbf{B}}\right)$ is "the right" equality. We are now in measure to close the conjecture. In fact, if we restrict our attention to systems, we can show that system contexts have the same discriminating power as arbitrary contexts.

Theorem 14 Let $M$ and $N$ be two systems, then $M \cong_{\mathbf{8}} N$ ifand only if $M \cong \cong_{\mathrm{p}} N$.

Proof $\quad M \cong_{\mathrm{p}} N$ implies $\mathrm{M} \cong_{\mathrm{s}} N$, by definition. For the converse, by Theorem 4 , if $M \cong_{\mathrm{s}}$ $N$ then $M \approx N$. As $\approx$ is preserved by system contexts, for all $n$ and $R n[M \mid R] \approx n[N \mid R]$. By Theorems 10 and 9 it follows that $M \cong \mathbf{p} N$.

This in turn implies a strong result: $\approx$ completely characterises $\cong_{\mathrm{p}}$ on systems.

Corollary 15 Let $M$ and $N$ be two systems, then $M \cong \cong_{\mathrm{p}} N$ if and only if $M \approx N$.

\section{Adding communication}

The basic idea is to have an output process such as $\langle E\rangle$, which outputs the message $E$, and an input process $(x) . Q$ which on receiving a message binds it to $x$ in $Q$ which then executes; here occurrences of $x$ in $Q$ are bound. Messages are sequences of capabilities. This form of message is more restrictive than those given in [5], but much of the power of name transmission can still be captured in our language. The syntax of our extended language is given in Table 6. We assume an understanding of free and bound variables, and of substitutions. A process $P$ is said to be closed if $\mathrm{fv}(P)=\emptyset$; otherwise is said to be open. The structural and reduction rules below define the semantics of communication:

$$
E .(F . P) \equiv(E . F) . P \quad \varepsilon . P \rightarrow P \quad(x) . P \mid\langle E\rangle \rightarrow P\{E / x\} .
$$

The LTS is extended by the introduction of two new pre-actions $(E)$ for input, $\langle-\rangle$ for output, and a new form of concretions $(\boldsymbol{\nu} \tilde{m})\langle E\rangle Q$. In Table 7 we give the rules that should be added to those of Table 3 and Table 4 to define the LTS $\boldsymbol{P} \stackrel{\boldsymbol{\alpha}}{\longrightarrow} \boldsymbol{P}^{\prime}$ for the closed processes of the extended calculus. Note that in the structural rules of Table 3 we are now assuming that parallel composition and restriction distribute over the new form of concretions $(\boldsymbol{\nu} \tilde{m})\langle E\rangle Q$ in the same manner as $(\boldsymbol{\nu} \tilde{m})\langle P\rangle Q$. The unusual preaction for output allows a uniform treatment of extrusion of names. Definition 3 and the extended LTS induce a bisimilarity relation, still denoted by $\approx$, over the closed systems of the message passing calculus.

For general terms, we define the open extension $\mathcal{R}^{\circ}$ of a relation $\mathcal{R}$ as: $P \mathcal{R}^{\circ} Q$ holds if and and only if for every closing substitution $\boldsymbol{\sigma}$ mapping from variables to expressions, we have $P \sigma \mathcal{R} Q \sigma$. 
Names: $\quad a, b, \ldots, k, l, m, n, \ldots \in \mathbf{N} \quad$ Systems:

Capabilities:

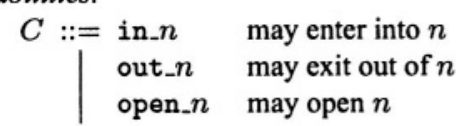

$$
M, N::=0 \quad \text { termination }
$$

parallel composition

$M_{1} \mid M_{2}$

$(\nu n) M \quad$ restriction

$n[P] \quad$ ambient

Expressions:

$$
\begin{aligned}
& E, F::=x \\
& \begin{array}{|ll}
C & \text { capability } \\
E . F & \text { path } \\
\varepsilon & \text { empty path }
\end{array}
\end{aligned}
$$

\section{Processes:}

$P, Q, R::=0$

\begin{tabular}{|l}
$P_{1} \mid P_{2}$ \\
$(\nu n) P$ \\
$G . P$ \\
$n[P]$ \\
$! G . P$ \\
$\langle E\rangle$
\end{tabular}

nil process

parallel composition

restriction

prefixing

ambient

replication

Guards:

$$
\begin{aligned}
G::=E & \text { expression } \\
\mid(x) & \text { input }
\end{aligned}
$$

output

Table 6. The Message-passing Mobile Ambients in Two Levels

$$
\begin{aligned}
& \text { Pre-actions: } \pi::=\ldots \\
& \text { (E) } \mid\langle-\rangle \\
& \text { Concretions: } K::=(\nu \tilde{m})\langle P\rangle Q \\
& \text { | }(\boldsymbol{\nu} \tilde{m})\langle E\rangle Q \\
& \frac{-}{\langle E\rangle \stackrel{(-)}{\longrightarrow}\langle E\rangle 0} \quad \frac{-}{(x) . P \stackrel{(E)}{\longrightarrow} P\{E / x\}} \quad \frac{E .(F . P) \stackrel{\pi}{\longrightarrow} Q}{(E . F) . P \stackrel{\pi}{\longrightarrow} Q} \\
& \frac{-}{\epsilon . P \stackrel{\tau}{\longrightarrow} P} \quad \frac{P \stackrel{\langle-\rangle}{\longrightarrow}(\nu \tilde{m})\langle E\rangle P^{\prime} \quad Q \stackrel{(E)}{\longrightarrow} Q^{\prime} \quad \text { fn }\left(Q^{\prime}\right) \cap\{\tilde{m}\}=\emptyset}{P \mid Q \stackrel{\tau}{\longrightarrow}(\nu \tilde{m})\left(P^{\prime} \mid Q^{\prime}\right)}
\end{aligned}
$$

Table 7. Pre-actions, Concretions and Labelled Transition System for Communication

Theorem 16 Relations $\approx^{\mathbf{0}}$ and $\cong_{\mathbf{8}}{ }^{\mathbf{0}}$ coincide over systems in the message-passing calculus.

Proof [Sketch] It is straightforward to extend Theorem 2 of [16] to the message-passing calculus. The completeness results follows because these relations are defined over systems and communication cannot be observed at top-level.

The open extension of the relation $\mathcal{S}$, written $\mathcal{S}^{\circ}$ can be shown equivalent to the relation

$$
\mathcal{S}^{\circ}=\left\{(P, Q): k[P \mid R] \approx^{\circ} k[Q \mid R], \text { for all } k, R \text { closed }\right\} .
$$

Our characterisation of reduction barbed equivalence over processes lifts smoothly to the message passing calculus.

Theorem 17 (Characterisation of $\cong_{p}^{\mathbf{e}}$ ) The relations $\cong_{\mathbf{p}}^{\mathrm{e}}$ and $\mathcal{S}^{\circ}$ coincide overprocesses in the message-passing calculus.

Proof [Sketch] It is easy to extend the proof of Theorem 10 to the closed terms of the message passing calculus. The result then follows from the definition of open extension.

The context lemma can be rephrased for the message passing calculus.

Theorem 18 Relations $\cong_{\mathbf{p}}^{\mathbf{0}}$ and $\cong_{\mathbf{p}}{ }^{\mathbf{o}}$ coincide overprocesses in the message-passing calculus. 
Proof The proof is an extension of the proof in the case without communication. We detail the case of closure under input prefix and replicated input prefix (for all the other cases it is enough to consider close terms).

Suppose that $P \simeq_{\mathrm{p}}^{\mathbf{e o}} Q$ and that $\mathrm{fn}(P) \cup \mathrm{fn}(Q) \subseteq\{x\}$. We want to show that $(x) \cdot P \cong \simeq_{\mathrm{p}}^{\mathbf{e}}$ $(x) \cdot Q$. For that we use our characterisation of $\simeq_{\mathrm{p}}^{\mathrm{e}}$ and we prove that for all $n, R$ closed it holds $n[(x) \cdot P \mid R] \approx n[(x) \cdot Q \mid R]$. In particular, we prove that the relation

$$
\mathcal{R}=\left\{(n[(x) \cdot P \mid R], n[(x) \cdot Q \mid R]): P \cong_{\mathrm{p}}^{\mathrm{oo}} Q, \mathrm{fn}(P, Q) \subseteq\{x\}, \forall n, R \text { closed }\right\}=\cup \approx
$$

is a bisimulation up to context and up to structural congruence. The most interesting case is when $n[(x) . P \mid R] \stackrel{\tau}{\longrightarrow} n\left[(\nu \tilde{r})\left(P\{E / x\} \mid R^{\prime}\right)\right] \equiv(\boldsymbol{\nu} \tilde{r}) n\left[P\{E / x\} \mid R^{\prime}\right]$, where $n \notin \tilde{r}$. Observe that $R$ sends the message $E$ and resumes as $R^{\prime}$. So we have a matching transition $n[(x) \cdot Q \mid R] \stackrel{\tau}{\longrightarrow} \equiv(\nu \tilde{r}) n\left[Q\left\{{ }^{E} / x\right\} \mid R^{\prime}\right]$. Since $P \cong_{\mathrm{p}}^{\otimes O} Q$, it holds $P\left\{{ }^{E} / x\right\} \cong_{\mathrm{p}}^{\mathrm{e}} Q\left\{{ }^{E} / x\right\}$. The characterisation of $\cong_{p}^{\mathbf{e}}$ guarantees that $n\left[P\left\{{ }^{E} / x\right\} \mid R^{\prime}\right] \approx n\left[Q\left\{{ }^{E} / x\right\} \mid R^{\prime}\right]$ and this allows us to conclude that up to context we are still in $\mathcal{R}$.

Suppose that $P \cong_{\mathrm{p}}^{\circ \circ} Q$ and that $\mathrm{fn}(P) \cup \mathrm{fn}(Q) \subseteq\{x\}$. Now we want to show that $!(x) . P \cong$ $!(x) \cdot Q$. Reasoning as before, we prove that for all $n, R$ closed it holds $n[!(x) \cdot P \mid R] \approx$ $n[l(x) \cdot Q \mid R]$. In particular, we prove that the relation

$$
\mathcal{R}=\left\{(n[!(x) \cdot P \mid R], n[!(x) \cdot Q \mid R)]: P \cong \cong_{p}^{\infty \circ} Q, \operatorname{fn}(P, Q) \subseteq\{x\}, \forall n, R \text { closed }\right\}=\cup \approx
$$

is a bisimulation up to context and up to $\gtrsim \approx$. The most interesting case is when $n[!(x), P \mid$ $R] \stackrel{r}{\rightarrow} n\left[(\boldsymbol{\nu} \tilde{r})\left(P\left\{{ }^{E} / x\right\}|!(x) . P| R\right)\right] \equiv(\nu \tilde{r}) n\left[P\left\{{ }^{E} / x\right\} \mid !(x) . P\right] \mid R^{\prime}$, where $n \notin \tilde{r}$ and $\tilde{r} \cap \operatorname{fn}(P)=\emptyset$. Observe that $R$ sends the message $E$ and resumes as $R^{\prime}$. So we have a matching transition $n ! !(x) \cdot Q \mid R] \stackrel{r}{\longrightarrow} \equiv(\nu \tilde{r}) n\left[Q\left\{{ }^{E} / x\right\}|!(x) \cdot Q| R^{\prime}\right]$, where $\tilde{r} \cap \mathrm{fn}(Q)=\emptyset$. By construction of $\mathcal{R}$ we have $n\left[P\left\{{ }^{E} / x\right\}|!(x) . P| R^{\prime}\right] \mathcal{R} n\left[P\left\{{ }^{E} / x\right\}|!(x) \cdot Q| R^{\prime}\right]$. Since $P \cong \cong_{\mathrm{p}}^{\ominus 0} Q$, it holds. $P\left\{{ }^{E} / x\right\} \cong_{\mathrm{p}}^{\theta} Q\left\{{ }^{E} / x\right\}$. The characterisation of $\cong_{\mathrm{p}}^{\circ}$ guarantees that $n\left[P\left\{{ }^{E} / x\right\} \mid\right.$ $\left\{(x) \cdot Q \mid R^{\prime}\right] \approx n\left[Q\left\{{ }^{E} / x\right\}|l(x) \cdot Q| R^{\prime}\right]$. Since bisimilarity is closed under restriction we have $(\boldsymbol{\nu} \tilde{r}) n\left[P\left\{{ }^{E} / x\right\}|!(x) \cdot Q| R^{\prime}\right] \approx(\boldsymbol{\nu} \tilde{r}) n\left[Q\left\{{ }^{E} / x\right\}|!(x) \cdot Q| R^{\prime}\right]$. This allows us to conclude that up to context (we factor out the context $(\boldsymbol{\nu} \tilde{\boldsymbol{r}})(-)$ ) and up to $\gtrsim, \approx$ we are still in $\mathcal{R}$.

Corollary 19 Relations $\mathcal{S}^{\mathbf{0}}$ and $\cong{ }_{\mathbf{p}}{ }^{\mathbf{c}}$ coincide overprocesses in the message-passing calculus.

A characteristic of working with systems deserves to be pointed out. Bisimilarity is defined over systems, and as such it cannot directly observe the exercise of communications capabilities (apart from internal communications). This allow us to avoid any special treatment for asynchronous communication. More than that, we can easily extend our results to a calculus equipped with synchronous communication (e.g., $\langle E\rangle, P)$.

\section{Algebraic theory}

In this section we give a collection of algebraic laws for $\cong_{p}$. First of all we recall the laws already proved in [16] with respect to $\cong_{8}$. By Theorem 14 these laws also hold for $\cong_{p}$ :

\section{Theorem 20 (System Laws)}

$$
\begin{aligned}
& I(\nu n) n[\langle E\rangle|(x) \cdot Q| M] \cong_{\mathrm{p}}(\nu n) n\left[Q\left\{{ }^{E} / x\right\} \mid M\right] \text { if } n \notin \mathrm{fn}(M) \\
& 2(\nu n) n\left[\langle E\rangle|(x) \cdot Q| \prod_{j \in J} \text { open_-kj, } R_{j}\right] \cong_{\mathrm{p}}(\nu n) n\left[Q\left\{{ }^{E} / x\right\} \mid \prod_{j \in J} \text { open_k } k_{j}, R_{j}\right] \\
& 3(\nu n) n[P] \cong_{\mathrm{p}} 0 \text { if } n \notin \mathrm{fn}(P)
\end{aligned}
$$


$4(\nu n)\left((\nu m) m\left[1 n \_n . P\right] \mid n[M]\right) \cong_{\mathrm{p}}(\nu n) n[(\nu m) m[P] \mid M]$ if $n \notin \operatorname{fn}(M)$

$s(\nu m, n)\left(m[\right.$ in_n.P $] \mid n\left[\prod_{j \in J}\right.$ open $\left.\left.k_{j} . R_{j}\right]\right) \cong_{\mathrm{p}}(\nu m, n) n\left[m[P] \mid \prod_{j \in J}\right.$ open_k $\left.k_{j} . R_{j}\right]$

$6(\nu n) n\left[(\nu m) m[\right.$ out_n.P] $\mid M] \cong_{p}(\nu n)((\nu m) m[P] \mid n[M])$ if $n \notin \mathrm{fn}(M)$

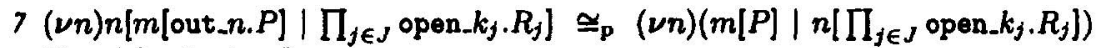
if $m \neq k_{j}$, for $j \in J$

$8 n[(\nu m)$ (open.m.P $\mid m[N]) \mid Q] \cong_{\mathrm{p}} n[(\nu m)(P \mid N) \mid Q]$ if $Q \equiv M \mid$ $\prod_{j \in J}(x) . R_{j}$ and $m \notin \mathrm{fn}(N)$

$9(\nu n) n[(\nu m)$ (open_m.P $\mid m[Q]) \mid R] \cong_{\mathrm{p}}(\nu n) n[(\nu m)(P \mid Q) \mid R]$ if $R \equiv$ $\prod_{i \in J}(x) . S_{i} \mid \prod_{j \in J}$ open $\_k_{j}, R_{j}$ and $m, n \notin \mathrm{fn}(Q)$

In Theorem 21 we give a collection of new algebraic laws involving processes. In Law 1 opening of private ambients containing arbitrary messages cannot be observed. Law 2 says that stuttering is not observable as well. Law 3 shows that processes prefixed by private capabilities are garbage. Law 4 says that two processes that differ only for having received different private capabilities cannot be distinguished. An instance of this law is

$$
(\nu n)\left\langle C_{n}\right\rangle \cong_{\mathrm{p}}(\nu n)\left\langle D_{n}\right\rangle
$$

for $C_{n}, D_{n} \in\{$ in_n, out_n, open_n\}. Notice that the above private outputs are not equivalent to $\mathbf{0}$. Law 5 is the Mobile Ambient variant of the asynchrony law [1] due to asynchronous communication. Finally, Law 6 equates two different outputs by adding a special process. While this law reminds us of Honda and Yoshida's equator [13], it should be pointed out that Honda and Yoshida's equators hide the difference between two channels, whereas we equate messages.

Theorem 21 (Process Laws)

$I(\nu n)\left(n\left[\prod_{j \in J}\left\langle E_{j}\right\rangle\right] \mid\right.$ open.n.P) $\cong_{\mathrm{p}} \prod_{j \in J}\left\langle E_{j}\right\rangle \mid P$ if $n \notin \mathrm{fn}\left(P, E_{j}\right)$ for all $j$

2 in_n.out_n.in_n.P $\cong_{\mathrm{p}}$ in_n.out_n.in_n.P $\oplus$ in_n.P where $\oplus$ is internal choice

$3(\nu n) C_{n} . P \cong_{\mathrm{p}} 0$ if $C_{n} \in\{$ in_n, out_n, open_n\}

$4(\nu n) P\left\{C_{n} / x\right\} \cong_{\mathrm{p}}(\nu n) P\left\{D_{n} / x\right\}$ if $\mathrm{fv}(P) \subseteq\{x\}$ and $n \notin \mathrm{fn}(P)$. for $C_{n}, D_{n} \in\{$ in_n, out_n, open_n $\}$

$s(x) \cdot(x) \cong_{\mathrm{p}} 0$

$6\langle E\rangle\left|E q(E, F) \cong_{\mathrm{p}}\langle F\rangle\right| E q(E, F)$ where $E q(E, F) \stackrel{\text { def }}{=} !(x) .\langle E\rangle \mid !(x) .\langle F\rangle$

Proof By Theorems 9 and 10, it suffices to show that

\section{$k[L H S \mid R] \approx k[R H S \mid R]$}

for all $k$ and $R$, where $L H S$ and $R H S$ denote the left hand side, right hand side, of each law. In all cases, except 4 , this can be proved by showing that the relation

$$
\mathcal{R}=\{(k|L H S| R], k[R H S \mid R]): \text { for all } k \text { and } R\}=\cup \mathcal{I}
$$

is a bisimulation up to context and up to $\gtrsim \approx$, where $\mathcal{I}$ represent the identity relation over systems.

In Law 4, the equality to prove is $k\left[(\nu n) P\left\{C_{n / x}\right\} \mid R\right] \approx k\left[(\nu n) P\left\{D_{n / x}\right\} \mid R\right]$, for all $k$ and $R$. This can be proved by showing that the relation

$$
\mathcal{R}=\left\{\left((\nu n) M\left\{C_{n / x}\right\},(\nu n) M\left\{D_{n / x}\right\}\right): \mathrm{fv}(M) \subseteq\{x\} \text { and } n \notin \mathrm{fn}(M)\right\}=
$$

is a bisimulation. Notice that, as $R$ is closed, up to $\alpha$-conversion, to avoid name-capturing, we have $k[(\nu n) P \sigma \mid R] \equiv(\nu n) k[P \mid R] \sigma$. 


\section{References}

[1] R. Amadio, I. Castellani, and D. Sangiorgi. On bisimulations for the asynchronous $\pi$ calculus. Theoretical Computer Science, 195:291-324, 1998.

[2] S. Arun-Kumar and M. Hennessy. An efficiency preorder for processes. Acta Informatica, 29:737-760, 1992.

[3] M. Bugliesi, S. Crafa, M. Merro, and V. Sassone. Communication interference in mobile boxed ambients. To appear in Information \& Computation 2004, an extended abstract appeared in Proc. FSTTCS'02, LNCS, Springer Verlag.

[4] L. Cardelli and A. Gordon. A commitment relation for the ambient calculus. 1996.

[5] L. Cardelli and A. Gordon. Mobile ambients. Theoretical Computer Science, 240(1): 177213, 2000. An extended abstract appeared in Proc. of FoSSaCS '98.

[6] G. Castagna and F. Zappa Nardelli. The seal calculus revisited: Contextual equivalence and bisimilarity. In Proc. 22nd FSTTCS '02, LNCS. Springer Verlag, 2002.

[7] G. Ferrari, U. Montanari, and E. Tuosto. A LTS semantics of ambients via graph synchronization with mobility. In Proc. ICTCS, LNCS, 2001.

[8] J.C. Godskesen, T. Hildebrandt, and V. Sassone. A calculus of mobile resources. In Proc. 10th CONCUR '02, LNCS, 2002.

[9] A. D. Gordon and L. Cardelli. Equational properties of mobile ambients. Journal of Mathematical Structures in CS, 12:1-38, 2002. Also in Proc. FoSSaCs ' 99.

[10] M. Hennessy, M. Merro, and J. Rathke. Towards a behavioural theory of access and mobility control in distributed system. In Proc. 5th FoSSaCS '03, LNCS, 2003.

[11] M. Hennessy, J. Rathke, and N Yoshida. safedpi: a language for controlling mobile code. In Proc. FOSSACS 03, LNCS, 2003.

[12] M. Hennessy and J. Riely. A typed language for distributed mobile processes. In Proc. 25th POPL. ACM Press, 1998.

[13] K. Honda and N. Yoshida. On reduction-based process semantics. Theoretical Computer Science, 152(2):437-486, 1995.

[14] F. Levi and D. Sangiorgi. Controlling interference in ambients. An extended abstract appeared in Proc. 27th POPL, ACM Press, 2000.

[15] M. Merro and M. Hennessy. Bisimulation congruences in safe ambients. In Proc. 29th POPL. ACM Press, 2002.

[16] M. Merro and F. Zappa Nardelli. Bisimulation proof methods for mobile ambients. In Proc. ICALP 2003, LNCS, Springer Verlag, 2003. An extended version is available as Computer Science Report 2003:01, University of Sussex.

[17] R. Milner, J. Parrow, and D. Walker. A calculus of mobile processes, (Parts I and II). Information and Computation, 100:1-77, 1992.

[18] D. Sangiorgi. Extensionality and intensionality of the ambient logic. In Proc. 28th POPL. ACMPress, 2001.

[19] A. Unyapoth and P. Sewell. Nomadic Pict: Correct communication infrastructures for mobile computation. In Proc. 28th POPL. ACM Press, 2001.

[20] M. G. Vigliotti. Transition systems for the ambient calculus. Master thesis, Imperial College of Science, Technology and Medicine (University of London), September 1999.

[21] J. Vitek and G. Castagna. Seal: A framework for secure mobile computations. In Internet Programming Languages, LNCS, pages 47-77. Springer Verlag, 1999. 